\title{
Sustained dual release of placental growth factor-2 and bone morphogenic protein-2 from heparin-based nanocomplexes for direct osteogenesis
}

This article was published in the following Dove Press journal:

International Journal of Nanomedicine

22 March 2016

Number of times this article has been viewed

\author{
Yun Liu, ${ }^{1, *}$ Li-Zhi Deng, ${ }^{2,3, *}$ \\ Hai-Peng Sun,' Jia-Yun \\ Xu, 'Yi-Ming Li,' Xin Xie,' \\ Li-Ming Zhang, ${ }^{2,3}$ Fei-Long \\ Deng'
}

'Department of Oral Implantology, Guangdong Provincial Key Laboratory of Stomatology, Guanghua School of Stomatology, Sun Yat-sen University, Guangzhou, People's Republic of China; ${ }^{2}$ PCFM Lab, ${ }^{3}$ GDHPPC Lab, Institute of Polymer Science, Department of Polymer and Materials Science, School of Chemistry and Chemical Engineering, Sun Yat-sen University, Guangzhou, People's Republic of China

*These authors contributed equally to this work
Objective: To compare the direct osteogenic effect between placental growth factor-2 (PlGF-2) and bone morphogenic protein-2 (BMP-2).

Methods: Three groups of PlGF-2/BMP-2-loaded heparin- $N$-(2-hydroxyl) propyl-3-trimethyl ammonium chitosan chloride (HTCC) nanocomplexes were prepared: those with $0.5 \mu \mathrm{g}$ PlGF-2; with $1.0 \mu \mathrm{g}$ BMP-2; and with $0.5 \mu \mathrm{g}$ PlGF-2 combined with $1.0 \mu \mathrm{g}$ BMP-2. The loading efficiencies and release profiles of these growth factors (GFs) in this nanocomplex system were quantified using enzyme-linked immunosorbent assay, their biological activities were evaluated using cell counting kit-8, cell morphology, and cell number counting assays, and their osteogenic activities were quantified using alkaline phosphatase and Alizarin Red S staining assays.

Results: The loading efficiencies were more than $99 \%$ for the nanocomplexes loaded with just PIGF-2 and for those loaded with both PIGF-2 and BMP-2. For the nanocomplex loaded with just BMP-2, the loading efficiency was more than $97 \%$. About $83 \%-84 \%$ of P1GF-2 and $89 \%-91 \%$ of BMP-2 were stably retained on the nanocomplexes for at least 21 days. In in vitro biological assays, PlGF-2 exhibited osteogenic effects comparable to those of BMP-2 despite its dose in the experiments being lower than that of BMP-2. Moreover, the results implied that heparin-based nanocomplexes encapsulating two GFs have enhanced potential in the enhancement of osteoblast function.

Conclusion: PlGF-2-loaded heparin-HTCC nanocomplexes may constitute a promising system for bone regeneration. Moreover, the dual delivery of PIGF-2 and BMP-2 appears to have greater potential in bone tissue regeneration than the delivery of either GFs alone.

Keywords: placental growth factor-2, bone morphogenic protein-2, heparin, nanocomplexes, osteogenesis

\section{Introduction}

Reconstruction of critical-sized bone defects remains a vitally important challenge. ${ }^{1-3}$ Its challenging nature is in part due to the development of bone tissue being a complex and coordinated process, involving direct and indirect osteogenesis. Indirect osteogenesis is mainly mediated by angiogenesis, which provides oxygen, nutrients, and pro-osteogenic cells to facilitate bone regeneration. ${ }^{4,5}$ Moreover, recent studies have suggested the existence of molecular crosstalk between angiogenesis and direct osteogenesis, which involves a myriad of growth factor (GF) cues. ${ }^{6}$

There has been increasing interest in mimicking the endogenous bone regeneration cascade by utilizing exogenous GFs, which play key roles in direct and indirect bone healing. ${ }^{2}$ Among the various direct osteogenic GFs, bone morphogenic protein-2 (BMP-2) is believed to be the most potent and has received clinical clearance from 
the US Food and Drug Administration..$^{7-9}$ However, a large dose of BMP-2 is necessary for an effective therapeutic effect because BMP-2 stimulates osteoregeneration only at a high concentration, ${ }^{10,11}$ and its half-life is as short as 7 minutes in vivo. ${ }^{11,12}$ This large dose results in high costs and adverse effects, including inflammation, swelling, ectopic bone formation, and even carcinogenicity. ${ }^{13}$ One feasible method to overcome these drawbacks is the selection of alternative GFs that enhance osteoregeneration at lower doses; another is the development of a GF delivery system that finds the ideal balance between releasing the GF at a minimal concentration and delivering it over a sufficiently long duration.

Placental growth factor (PlGF) was originally discovered as an angiogenic GF belonging to the vascular endothelial growth factor (VEGF) family and plays a potent role in angiogenesis. ${ }^{14-18}$ Although it is well established that VEGFs enhance osteogenesis indirectly and directly, little is known about PlGF. ${ }^{16,17}$ While human Plgf gene encodes four isoforms (PlGF1-4), mouse Plgf gene encodes a single isoform (PlGF2). ${ }^{19}$ There has also been an increasing interest in PIGF-2 during the past few years. Recent studies have implied that PlGF-2 is a multitasking cytokine that stimulates and activates numerous cell types, including bone marrow-derived cells. ${ }^{14,19-21}$

Apart from its traditional role as an angiogenic GF, PlGF-2 also plays an important role in bone remodeling and regeneration. ${ }^{10,18}$ Blocking of $\mathrm{PlGF}-2$ receptors could inhibit bone formation in vivo. ${ }^{10,22,23}$ Moreover, some in vitro studies confirmed that PlGF-2 directly enhances markers of bone regeneration in osteoblasts and also enhances osteoclast migration and differentiation. ${ }^{10,24-27}$ A recent study by McCoy et $\mathrm{al}^{10}$ indicated that PlGF-2 plays a concentration-dependent osteogenic role different from that of BMP-2. Intriguingly, in contrast to BMP-2, which at higher concentrations induces more obvious bone regeneration, ${ }^{13} \mathrm{PlGF}-2$ acts in a more complex fashion. Briefly, while relatively high concentrations of PlGF-2 enhance angiogenesis and osteoclast recruitment and activation, low concentrations exhibit a direct osteogenic effect. ${ }^{10}$ The mechanism underlying these concentration effects could be associated with the mechanical stimulation and the activation of hypoxia-mediated pathways. ${ }^{10,28}$ Moreover, the PlGF-2 gene expression levels correlate with the duration and magnitude of stimulation, providing a potent mechanism by which PlGF-2 modulates osteogenesis in a concentration-dependent fashion. ${ }^{10}$

For this study, we focused on the direct osteogenic effect of PlGF-2. We used MC3T3-E1 cells as the model cell line for in vitro osteogenesis analysis, which has been proven to be effective. ${ }^{29}$ PlGF $(0.5 \mu \mathrm{g})$ was loaded at half of the dose of BMP-2 $(1.0 \mu \mathrm{g})$ to investigate whether the PlGF-2 loaded at such a lower dose would exhibit an osteogenic effect comparable to the BMP-2 loaded at the higher dose. Furthermore, we used nanocomplexes loaded with both GFs $(0.5 \mu \mathrm{g}$ P1GF-2/1.0 $\mu \mathrm{g}$ BMP-2) to investigate any enhanced potential in bone tissue regeneration. An outline of this study is illustrated in Figure 1.

\section{Materials and methods Materials}

Heparin sodium (molecular weight: 6,000-15,000 Da) was supplied by Qiyun Biotechnology Company Ltd (Guangzhou, People's Republic of China). Chitosan (with a viscosity of less than $200 \mathrm{mPa} \cdot \mathrm{s}$ ) was supplied by the Jingchun Biochemical Technology Company Ltd (Shanghai, People's Republic of China). Glycidyl trimethyl ammonium chloride and Alizarin Red S (ARS) were supplied by the Sigma Chemical Company (Darmstadt, Germany). Human recombinant BMP-2 (Catalog 120-02/Lot 0411255) and human recombinant PlGF-2 (Catalog 100-56/Lot 0908460-1) were purchased from PeproTech (Rocky Hill, NJ, USA) and their uses were approved by Guanghua School of Stomatology at Sun Yat-sen University. Enzyme-linked immunosorbent assay (ELISA) kits for BMP-2 and PlGF-2 were purchased from CloudClone Corp (Houston, TX, USA). The osteoblast-like cell line MC3T3-E1 (subclone 14) was obtained from the Shanghai
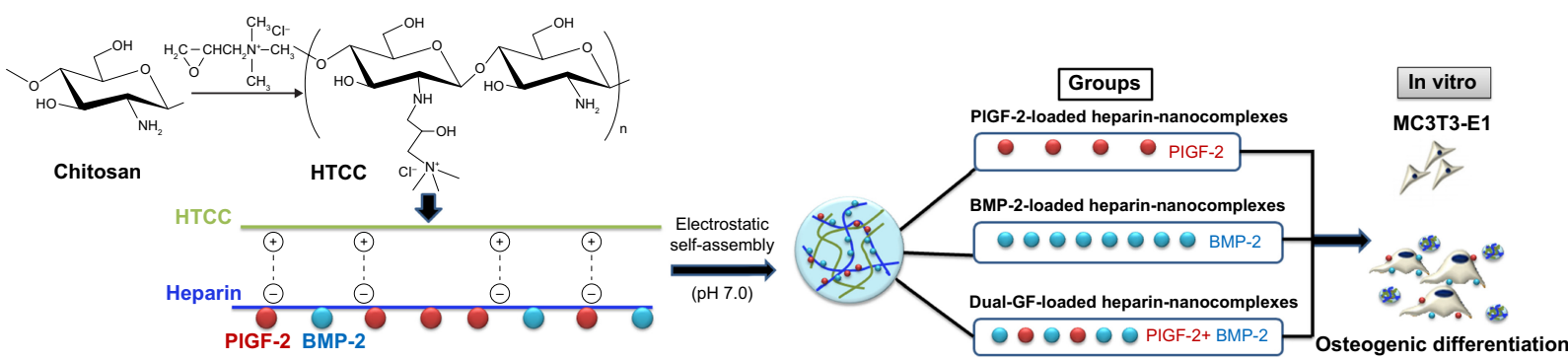

Figure I Schematic illustration of the fabrication and in vitro osteogenic effect of PIGF-2-/BMP-2-loaded heparin-HTCC nanocomplexes. Abbreviations: PIGF-2, placental growth factor-2; BMP-2, bone morphogenetic protein; GF, growth factor; HTCC, N-(2-hydroxyl)propyl-3-trimethyl ammonium chitosan chloride. 
Cellular Institute of the China Scientific Academy (Shanghai, People's Republic of China). $\alpha$-Minimum essential medium $(\alpha-\mathrm{MEM})$ and a combined antimycotic antibiotic solution was obtained from Gibco (Carlsbad, CA, USA), while fetal calf serum was obtained from Hycolone (Logan, UT, USA). Cell counting kit-8 (CCK-8), used for the cell proliferation assay, was sourced from Dojindo (Kumamoto, Japan). An alkaline phosphatase (ALP) assay kit was obtained from Jiancheng (Nanjing, People's Republic of China) and a bicinchoninic acid protein assay kit from Beyotime (Shanghai, People's Republic of China). A Sunrise ELISA reader was obtained from Tecan (Zurich, Switzerland).

\section{Preparation and characterization of heparin-based nanocomplexes}

A water-soluble chitosan derivative, $N$-(2-hydroxyl) propyl3-trimethyl ammonium chitosan chloride (HTCC), was synthesized as described previously. ${ }^{29,30}$ To prepare heparinHTCC nanocomplexes, heparin and HTCC powder were first dissolved in deionized water to obtain a final concentration of $2 \mathrm{mg} / \mathrm{mL}$ of each. Then, the two aqueous solutions were sterilized by passing them through $0.22 \mu \mathrm{m}$ syringe filters, followed by adding the heparin solution dropwise to the HTCC solution (volume ratio of heparin:HTCC $=3: 5$ ) with continuous stirring. Fifteen minutes later, the total $20 \mathrm{~mL}$ of the nanocomplex suspension was transferred into a dialysis bag with a molecular weight cut-off of 300,000 Da. The dialysis bag was then immersed in $50 \mathrm{~mL}$ of simulated body fluid and incubated in a reciprocal shaking water bath at $37^{\circ} \mathrm{C}$. At predetermined time intervals (days 1, 3, 7, and 14), $3 \mathrm{~mL}$ of each of the nanocomplex suspensions was taken out for further tests. Briefly, the particle sizes and zeta potentials of various nanocomplexes were measured with Zeta PALS instrument (Brookhaven Inst. Ltd, Holtsville, NY, USA), while the morphologies were visualized by using transmission electron microscopy (TEM).

\section{Preparation and characteristics of heparin-HTCC nanocomplexes loaded with PIGF-2 and/or BMP-2}

PlGF-2 and BMP-2 were localized by adding $0.5 \mu \mathrm{g}$ PlGF- 2 or $1.0 \mu \mathrm{g}$ BMP-2 or a combination of $0.5 \mu \mathrm{g}$ PlGF-2 and $1.0 \mu \mathrm{g}$ BMP-2 to $1.25 \mathrm{~mL}$ of $2 \mathrm{mg} / \mathrm{mL}$ heparin in Dulbecco's phosphate-buffered saline (DPBS), for binding. Three mixtures were produced by gently stirring these components for 12 hours at $4^{\circ} \mathrm{C}$, and each of these mixtures was then added dropwise to $2.15 \mathrm{~mL}$ of HTCC solution (2 $\mathrm{mg} / \mathrm{mL}$ in DPBS) under continuous stirring (volume ratio of heparin:HTCC $=3: 5$ ).
The resulting mixtures were then subjected to centrifugation for 5 minutes at $12,000 \times g$ to collect the respective GF-loaded heparin-HTCC nanocomplexes. The supernatant was collected to determine the amount of GF (PlGF-2 and/or BMP-2) loaded using ELISA kits (according to the manufacturer's instructions). In each case, the GF loading efficiency was determined by applying the following equation: ${ }^{30}$

$$
\underset{\text { efficiency }(\%)}{\text { Loading }}=\frac{\text { Total amount of GF }- \text { Free GF }}{\text { Total amount of GF added }} \times 100 \% \text {. }
$$

The compositions of the nanocomplex groups are summarized in Table 1. The morphologies and stabilities of the nanocomplexes were analyzed by visualizing them using TEM and measuring their zeta potentials.

\section{In vitro PIGF-2/BMP-2 release}

In vitro release of PlGF-2/BMP-2 from the GF-loaded nanocomplexes was carried out in $\alpha$-MEM complete medium. Different heparin-HTCC mixtures $(3.4 \mathrm{~mL})$ were put in the inner filter concentrator tubes of ultrafiltration centrifugal tubes (Millipore's Amicon ${ }^{\circledR}$ Ultra-15, Millipore, Billerica, MA, USA, with a cut-off molecular weight of 300,000 Da), and then the inner tubes were immersed in the outer tubes containing $8 \mathrm{~mL} \alpha$-MEM complete medium. Each sample was then incubated with a mild mechanical stirring/shaking motion $(50 \mathrm{rpm})$ at $37^{\circ} \mathrm{C}$. At each predetermined time point over the course of 21 days, the medium in the outer centrifuge tubes was withdrawn and replenished with fresh $\alpha$-MEM complete medium. The amount of PlGF-2/BMP-2 in the medium was determined using ELISA kits, according to the manufacturer's instructions. The medium collected at various time points is referred to as "released medium". This released medium was frozen and stored at $-80^{\circ} \mathrm{C}$ until used in the in vitro assays.

\section{MC3T3-EI cell culture}

The MC3T3-E1 preosteoblast cells (subclone 14, obtained from the Shanghai Cellular Institute of the China Scientific

Table I Nanocomplex groups and their compositions

\begin{tabular}{lll}
\hline Groups & $\begin{array}{l}\text { PIGF-2 } \\
\text { content }(\mu \mathbf{g})\end{array}$ & $\begin{array}{l}\text { BMP-2 } \\
\text { content }(\mu \mathbf{g})\end{array}$ \\
\hline Blank heparin-nanocomplexes & 0 & 0 \\
PIGF-2-loaded heparin-nanocomplexes & 0.5 & 0 \\
BMP-2-loaded heparin-nanocomplexes & 0 & 1.0 \\
Dual-GF-loaded heparin-nanocomplexes & 0.5 & 1.0 \\
\hline
\end{tabular}

Abbreviations: PIGF-2, placental growth factor-2; BMP-2, bone morphogenetic protein; GF, growth factor. 
Academy, Shanghai, People's Republic of China) used in our experiments ranged from passages 8 to 20. Cells were cultured in regular culture media consisting of $\alpha$-MEM medium supplemented with $10 \%$ fetal calf serum and $1 \%$ antibiotic/ antimycotic solution. The MC3T3-E1 cells were incubated at $37^{\circ} \mathrm{C}$ in an incubator containing $5 \% \mathrm{CO}_{2}$.

\section{In vitro bioactivity test}

The in vitro bioactivity of the released PlGF-2/BMP-2 from heparin-HTCC nanocomplexes collected at different times was tested by an MC3T3-E1 proliferation assay and osteogenesis assay.

\section{In vitro cell viability assay}

The influence of the released PlGF-2/BMP-2 on the proliferation of MC3T3-E1 cells was determined by a CCK-8 assay, using a standard protocol. ${ }^{31}$ In brief, MC3T3-E1 cells were seeded in 96-well plates at a density of $3 \times 10^{3}$ cells/ well and incubated overnight in $5 \% \mathrm{CO}_{2}$ at $37^{\circ} \mathrm{C}$. Then the medium was replaced with "released medium" prepared as described in the section "In vitro P1GF-2/BMP-2 release". The medium was changed every 2 days. At days 1 and 3, cell viabilities were examined using $\mathrm{CCK}-8$ assays. At the scheduled time points, $10 \mu \mathrm{L}$ of CCK-8 was added to each well. Then the cells were incubated for another 2 hours at $37^{\circ} \mathrm{C}$, and the optical density (OD) value was measured at an optical wavelength of $450 \mathrm{~nm}$ (ELISA reader).

\section{In vitro cell spreading and proliferation assays}

For cell spreading and proliferation examinations, the morphologies of the MC3T3-E1 cells cultured with various "released medium" were observed directly by using an inverted phase contrast microscope (Axio observer Z1, Zeiss, Oberkochen, Germany). Briefly, the cells were seeded in 24-well plates at a density of $1.6 \times 10^{4}$ cells/well and incubated overnight in $5 \% \mathrm{CO}_{2}$ at $37^{\circ} \mathrm{C}$. In parallel, the same culture conditions as for the cell viability assays were applied to the MC3T3-E1 cells. After 12 hours and 3 days, the images of the cells were obtained for analysis, respectively. Furthermore, the cell numbers were determined by a trypsinization method, according to a previously published procedure. ${ }^{32}$ After culturing for 1 and 3 days, the cells cultured with the various "released medium" were rinsed with PBS, and then detached by trypsinization and counted using hemocytometers. To ensure representative cell counts, three replicates in each group were performed.

\section{In vitro ALP activity assay}

The osteogenic bioactivity of the released PlGF-2/BMP-2 was determined by measuring its ability to stimulate ALP activity. Briefly, the MC3T3-E1 cells were seeded in 24-well plates at a density of $1 \times 10^{4}$ cells/well and incubated overnight in $5 \% \mathrm{CO}_{2}$ at $37^{\circ} \mathrm{C}$. Then, osteogenic medium (the released medium supplemented with $10 \mathrm{mM} \beta$-glycerol phosphate, $0.05 \mathrm{mM}$ L-ascorbic acid, and $100 \mathrm{mM}$ dexamethasone) was applied. After 7 and 10 days of culture, the ALP activity was measured by using an ALP activity assay kit (Jiancheng), according to the standard procedures, ${ }^{33}$ and was then normalized to the total protein content, which was measured with a protein assay kit (Beyotime).

\section{In vitro ARS staining and quantification}

In parallel with the ALP assay, ARS staining was performed to detect the mineralization effect of the released PlGF-2/ BMP-2. MC3T3-E1 cells were seeded in 6-well plates at a density of $1 \times 10^{5}$ cells/well and cultured in $5 \% \mathrm{CO}_{2}$ at $37^{\circ} \mathrm{C}$. After 24 hours, the medium was replaced with an osteogenic medium (the released medium supplemented with $10 \mathrm{mM}$ $\beta$-glycerol phosphate, $0.05 \mathrm{mM}$ L-ascorbic acid, and $100 \mathrm{mM}$ dexamethasone; and in this assay the released medium was collected at day 7). The culture medium was changed every 3 days. At the end of the 21-day incubation period, the mineralization effect was detected by ARS staining. In brief, the MC3T3-E1 cells were washed with PBS and fixed with $4 \%$ formaldehyde for 15 minutes. Then, the fixed cells were stained with $2 \%$ Alizarin Red ( $\mathrm{pH} 4.2$ ) for 5 minutes at room temperature. The cells were then rinsed with PBS three times and viewed with an inverted light microscope (Axio observer Z1, Zeiss). In addition, the calcium mineral contents were quantified by a destaining method as previously described. ${ }^{34,35}$ The stained samples were extracted with $10 \%$ $(\mathrm{v} / \mathrm{v})$ acetic acid for 30 minutes at room temperature. The solubilized ARS extracts were then transferred to a 96-well plate, and the OD values were measured at $560 \mathrm{~nm}$ (ELISA reader). ${ }^{36}$

\section{Statistical analysis}

All quantitative data are shown as mean \pm standard deviation. Statistical comparisons were carried out by using one-way analysis of variance, followed by Tukey's test for multiple comparisons. The significance value was set at $P<0.05$. All data were analyzed with SPSS 21.0 software (IBM Corporation, Armonk, NY, USA). 


\section{Results and discussion Loading of PIGF-2/BMP-2}

Three different combinations of GFs, specifically $0.5 \mu \mathrm{g}$ PIGF-2 with $1.0 \mu \mathrm{g}$ BMP-2 (so-called "dual-GF loaded"), $1.0 \mu \mathrm{g}$ BMP-2 without PlGF-2, and $0.5 \mu \mathrm{g}$ PlGF-2 without BMP-2, as well as a GF-free sample, were immobilized in heparin-HTCC nanocomplexes (Table 1). For BMP-2, less than $3 \%$ of this GF was detected by using ELISA in the supernatant immediately after forming the nanocomplexes, confirming a high loading efficiency. And for PIGF-2, less than $1 \%$ was detected, indicating an even higher loading efficiency (Table 2). These high-efficiency values can be explained by the high binding affinity of the negatively charged heparin for positively charged GFs such as PlGF-2 and BMP-2 ${ }^{30,37-42}$ Furthermore, heparin can specifically interact with heparin-binding GFs. ${ }^{38,39,43}$ Also, Martino et al ${ }^{40}$ showed that domain II (Fg $\left.\beta 15-66_{(2)}\right)$ of heparin exhibits high affinity for GFs in general, but with heparin displaying a fourfold stronger affinity for PlGF-2 than for BMP-2; ${ }^{44}$ they also revealed a specific heparin-binding portion of PlGF-2 spanning residues 123-144 and having the sequence RRPKGRGKRRREKQRPTDCHL. ${ }^{44}$ However, in our study, the difference of loading efficiency between PlGF-2 and BMP-2 was not so prominent, perhaps because both GFs were applied at overall low doses and exhibited high affinity to heparin. Also, the zeta potential and TEM test (Table 3, Figures 2 and S1) revealed that the average nanocomplex diameter increased after loading a single GF or both GFs together, and, intriguingly, that the immobilization of GFs was associated with gains in zeta potential and diameter of the nanocomplexes.

\section{Sustained release of PIGF-2/BMP-2}

For all the nanocomplexes, minimal burst releases of less than $3 \%$ were observed after 1 day (Figure 3 ). Thereafter, the release profiles were linear and steep until day 7 , after which the release rates slowed and became more sustainable. After

Table 2 Loading efficiency of PIGF-2/BMP-2 into heparin-HTCC nanocomplexes

\begin{tabular}{lll}
\hline Group & $\begin{array}{l}\text { PIGF-2 loading } \\
\text { efficiency (\%) }\end{array}$ & $\begin{array}{l}\text { BMP-2 loading } \\
\text { efficiency (\%) }\end{array}$ \\
\hline PIGF-2-loaded heparin-nanocomplexes & 99.81 & - \\
BMP-2-loaded heparin-nanocomplexes & - & 97.79 \\
Dual-GF-loaded heparin-nanocomplexes & 99.79 & 98.93
\end{tabular}

Abbreviations: PIGF-2, placental growth factor-2; BMP-2, bone morphogenetic protein; HTCC, N-(2-hydroxyl)propyl-3-trimethyl ammonium chitosan chloride; $\mathrm{GF}$, growth factor.
Table 3 Characteristics of heparin-HTCC nanocomplexes before and after GF loading

\begin{tabular}{|c|c|c|c|c|}
\hline \multirow[t]{2}{*}{ Group } & \multirow[t]{2}{*}{ PDI } & \multirow{2}{*}{$\begin{array}{l}\text { Zeta } \\
\text { potential } \\
(\mathrm{mV})\end{array}$} & \multicolumn{2}{|c|}{ Mean diameter $(\mathrm{nm})$} \\
\hline & & & $\begin{array}{l}\text { From zeta } \\
\text { potential test }\end{array}$ & $\begin{array}{l}\text { From } \\
\text { TEM }\end{array}$ \\
\hline $\begin{array}{l}\text { Blank heparin- } \\
\text { nanocomplexes }\end{array}$ & $0.10 \pm 0.02$ & $31.3 \pm 1.6$ & $371.9 \pm 4.8$ & 213.0 \\
\hline $\begin{array}{l}\text { PIGF-2-loaded heparin- } \\
\text { nanocomplexes }\end{array}$ & $0.11 \pm 0.03$ & $37.4 \pm 0.3$ & $539.2 \pm 6.4$ & 298.1 \\
\hline $\begin{array}{l}\text { BMP-2-loaded heparin- } \\
\text { nanocomplexes }\end{array}$ & $0.13 \pm 0.03$ & $34.5 \pm 0.8$ & $406.0 \pm 5.1$ & 244.7 \\
\hline $\begin{array}{l}\text { Dual-GF-loaded } \\
\text { heparin-nanocomplexes }\end{array}$ & $0.16 \pm 0.02$ & $35.1 \pm 0.3$ & $485.7 \pm 6.2$ & 266.4 \\
\hline
\end{tabular}

Note: Data are presented as mean \pm standard deviation.

Abbreviations: HTCC, N-(2-hydroxyl)propyl-3-trimethyl ammonium chitosan chloride; GF, growth factor; PDI, polydispersity index; PIGF-2, placental growth factor-2; BMP-2, bone morphogenetic protein; TEM, transmission electron microscopy.

21 days, less than $17 \%$ of the PlGF- 2 was released from the nanocomplexes, while for BMP-2, the release percentage was less than $12 \%$. These data indicate that the nanocomplexes can efficiently control the release of PlGF-2 and BMP-2. Moreover, the nanocomplexes loaded with single GFs presented slightly faster release profiles than did those loaded with both GFs. In addition, BMP-2 was released a bit more slowly than PlGF-2. On day 21, the cumulative amounts of BMP-2 released from nanocomplexes loaded with just BMP-2 and from those loaded with both BMP-2 and PIGF-2 were 11\% ( 110 $\mathrm{ng}$ ) and 9\% ( 90 ng), respectively, while the cumulative amounts of PlGF-2 released from nanocomplexes loaded with just PIGF-2 and from the dual-GF-loaded nanocomplexes were $17 \%(\sim 85 \mathrm{ng})$ and $16 \%(\sim 80 \mathrm{ng})$, respectively. All these results indicated that sustained deliveries of PIGF-2 and BMP-2 can be achieved by the heparin-HTCC nanocomplexes. Intriguingly, BMP-2 was released from the dual-GF-loaded nanocomplexes in a more constant manner than PIGF-2, indicative of sequential-release-like kinetics. According to recent research, PlGF-2 plays the role of a multitasking cytokine and stimulates bone regeneration by direct and indirect mechanisms - and this GF also has different effects at different concentrations, enhancing vascularization at relatively higher concentrations while enhancing osteogenic differentiation at lower concentrations. ${ }^{10}$ As the source of nutrients, oxygen and stem cells, vasculature is critical for osteogenesis. ${ }^{45}$ Accordingly, the sequential release kinetics indicated in this study may apply: in brief, the nanocomplexes release more PlGF-2 in the early phase to enhance angiogenesis; then, the subsequently released PlGF-2, at this point at lower concentrations than BMP-2, enhances direct osteogenesis in coordination with BMP-2. 
A

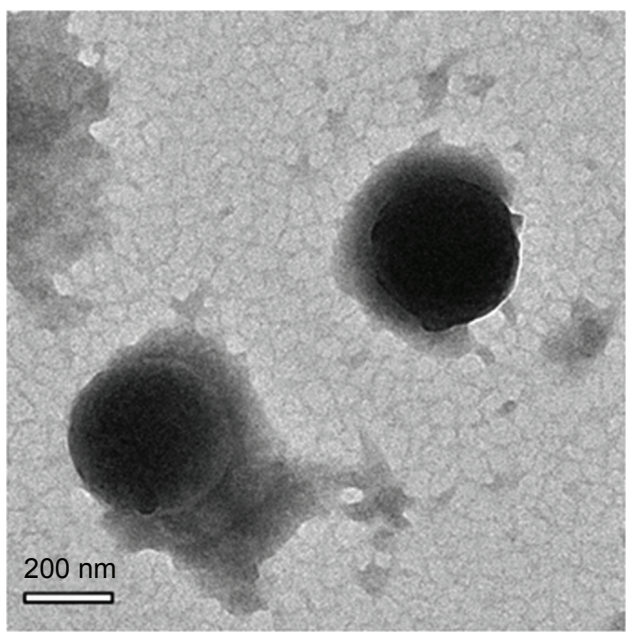

B

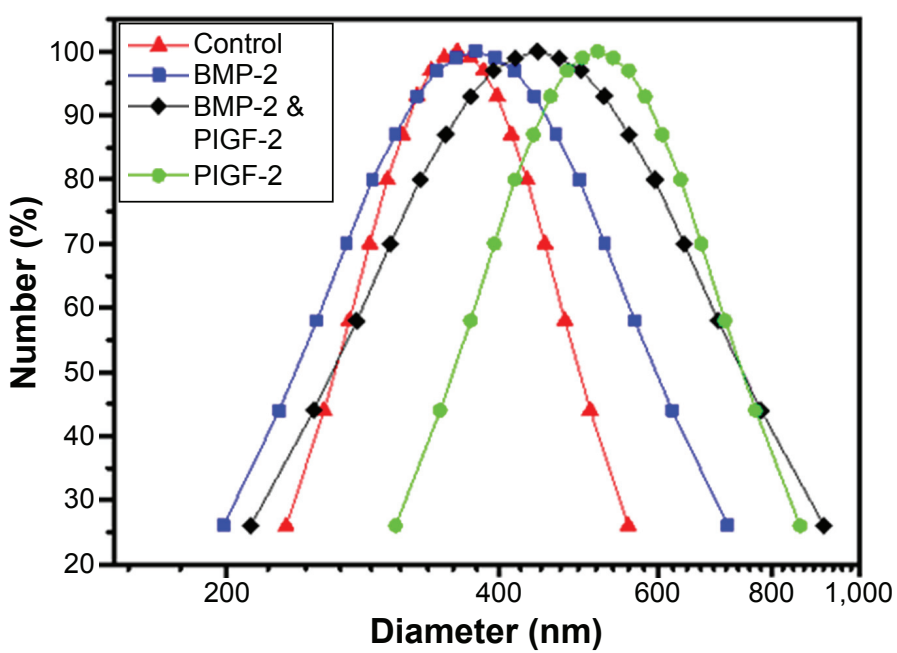

Figure 2 (A) TEM images of heparin-HTCC nanocomplexes after dual GF loading (scale bar =200 nm), (B) size distribution of heparin-HTCC nanocomplexes before and after PIGF-2/BMP-2/dual-GF loading.

Abbreviations: BMP-2, bone morphogenetic protein; PIGF-2, placental growth factor-2; TEM, transmission electron microscopy; HTCC, N-(2-hydroxyl)propyl-3-trimethyl ammonium chitosan chloride; GF, growth factor.

In this study, heparin acts as a "sandwich" or "double duty" component and interacts with HTCC to form highly stable polyelectrolyte complexes ${ }^{37}$ that have been demonstrated to effectively load GFs and maintain their bioactivities. ${ }^{13,30,44,46}$ The minimal initial release may be attributed to the release of GFs that were physically adsorbed onto the heparin-HCCT nanocomplexes. The degradation test proved the stability of the nanocomplexes (Table 4, Figure 4), and its results matched the sustained release pattern. BMP-2 exhibited a more constant release than did PlGF-2, not only from the

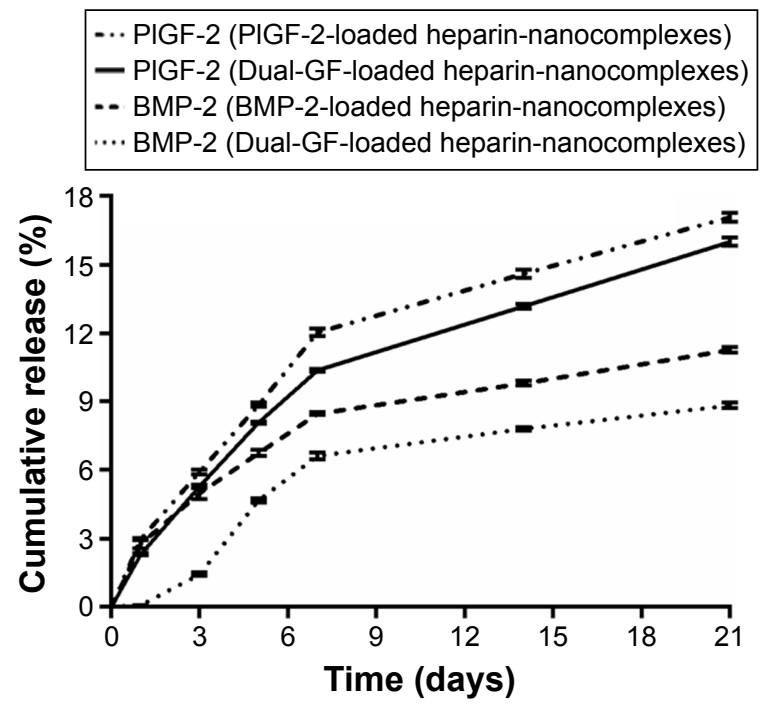

Figure 3 Release profiles of PIGF-2/BMP-2 from heparin-HTCC nanocomplexes. Abbreviations: PIGF-2, placental growth factor-2; GF, growth factor; BMP-2, bone morphogenetic protein; HTCC, N-(2-hydroxyl)propyl-3-trimethyl ammonium chitosan chloride. aforementioned dual-GF-loaded nanocomplexes but also from the single-GF-loaded nanocomplexes. This phenomenon can be largely explained by the different GF doses. As the PIGF-2 dose was just half that of BMP-2, we hypothesize that the PlGF-2 molecules were distributed farther apart from each other than were BMP-2 molecules, which would cause the release of PIGF-2 to be easier and faster. This hypothesis has yet to be tested.

\section{In vitro bioactivity test}

The medium released from the GF-loaded nanocomplexes induced significantly greater CCK- 8 activities than did that released from the blank nanocomplexes (Figure 5), indicating that the released PlGF-2/BMP-2 from heparin/ HTCC nanocomplexes maintained their activities. Besides that, the results indicated that the PlGF-2, despite its lower dose, exhibited a similar level of CCK-8 activity as did the BMP-2. Also, compared to the other medium, the medium released from the dual-GF-loaded nanocomplexes yielded a significantly greater activity, indicating a greater potential to enhance the proliferation rate of MC3T3-E1 cells.

Table 4 Stability test for heparin-HTCC nanocomplexes

\begin{tabular}{llll}
\hline Day & Mean diameter $(\mathbf{n m})$ & PDI & Zeta potential $(\mathbf{m V})$ \\
\hline $\mathrm{I}$ & $371.9 \pm 4.8$ & $0.10 \pm 0.02$ & $31.3 \pm 1.6$ \\
3 & $363.2 \pm 5.1$ & $0.09 \pm 0.02$ & $32.1 \pm 1.3$ \\
7 & $351.2 \pm 6.2$ & $0.11 \pm 0.03$ & $33.4 \pm 1.1$ \\
14 & $319.3 \pm 6.4$ & $0.12 \pm 0.02$ & $35.0 \pm 0.8$ \\
\hline
\end{tabular}

Note: Data are presented as mean \pm standard deviation.

Abbreviations: HTCC, N-(2-hydroxyl)propyl-3-trimethyl ammonium chitosan chloride; PDI, polydispersity index. 


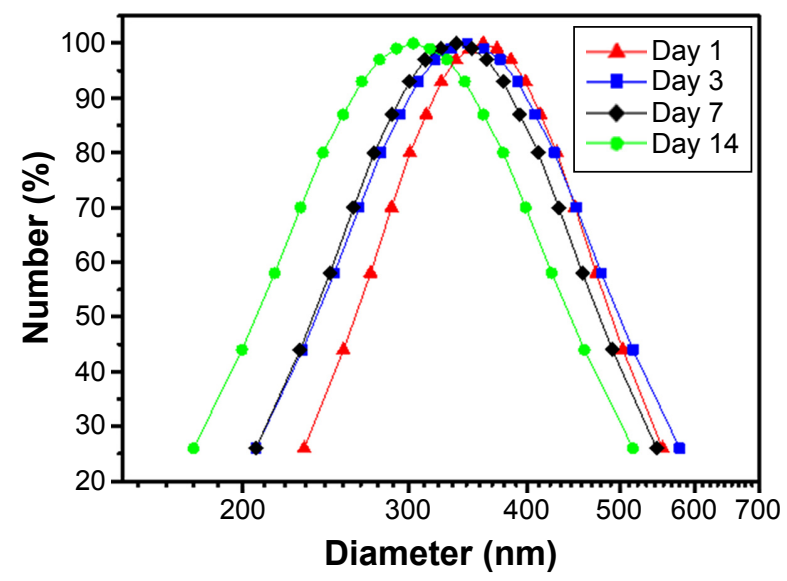

Figure 4 Degradation test of heparin-HTCC nanocomplexes tested in SBF for various days.

Abbreviations: HTCC, N-(2-hydroxyl)propyl-3-trimethyl ammonium chitosan chloride; SBF, simulated body fluid.

We also used an inverted phase-contrast microscope to assess the morphologies of MC3T3-E1 cells cultured with various release mediums for 12 and 72 hours, respectively. At each time point, there was major cell growth for the dualGF-loaded nanocomplex group (Figure 6[c]), while the blank nanocomplex group showed the fewest cells (Figure 6[d]). The MC3T3-E1 cells for all the groups proliferated stably

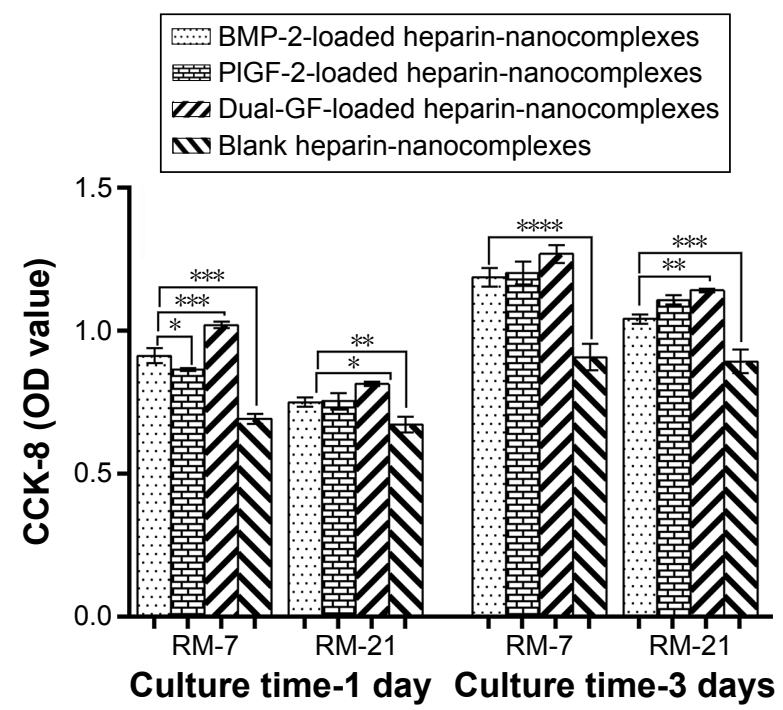

Figure 5 MC3T3-EI proliferation with the PIGF-2-/BMP-2-loaded heparin-HTCC nanocomplexes.

Notes: Released medium collected from various nanocomplexes was used to culture MC3T3-EI cells for 96 hours. The CCK-8 assay was performed on days I and 3 postseeding to determine the viability of the cells cultured with the released GFs. The released medium was collected on days 7 and 21 for analysis. Blank nanocomplexes were defined as the control. Error bars represent SD. $* P<0.05$, $* * p<0.01$, *** $P<0.001$, **** $P<0.000$ I.

Abbreviations: BMP-2, bone morphogenetic protein; PIGF-2, placental growth factor-2; GF, growth factor; CCK-8, cell counting kit-8 assay; OD, optical density; RM, released medium; RM-7, released medium collected on the seventh day; RM$2 \mathrm{I}$, released medium collected on the 2 Ist day; HTCC, $\mathrm{N}$-(2-hydroxyl)propyl-3trimethyl ammonium chitosan chloride; SD, standard deviation. with incubation, and the cells were characteristically spindle shaped (Figure 6A and B). The MC3T3-E1 cells for dualGF-loaded nanocomplex group expanded even more and yielded an even and dense MC3T3-E1 cell monolayer after 3 days in culture (Figure 6A and B[c]), while the MC3T3-E1 cells for single-GF-loaded nanocomplexes showed flat fibroblast-like morphologies, but did not reach confluence (Figure $6 \mathrm{~A}$ and $\mathrm{B}[\mathrm{a}, \mathrm{b}]$ ). The growth of MC3T3-E1 cells for different groups was further assessed by cell number counting (Figure 6C), and a similar trend was observed. These results demonstrated that the dual-GF-loaded nanocomplexes presented better microenvironments for the proliferation and expansion of MC3T3-E1 cells than did the single-GF-loaded nanocomplexes.

In addition, the ALP activity was evaluated after 7 and 10 days of culture, which is a well-established method to confirm having reached the initial stages of osteoblast-like cells differentiation. ${ }^{47}$ The ALP activity increased gradually in a time-dependent manner (Figure 7). Although the ALP activity of the blank nanocomplex group was significantly lower than that of the others, there were no significant differences among the three GF-loaded nanocomplex groups on day 7. However, on day 10, the dual-GF-loaded nanocomplex group exhibited significantly higher ALP activity than did the single-GF-loaded nanocomplex groups. In addition, the difference between the PIGF-2-loaded nanocomplex group and BMP-2-loaded nanocomplex group was not significant. These results were similar to the calcium assay.

In parallel, we investigated the mineral deposition both qualitatively and quantitatively (Figure 8). ARS assay showed that all the groups demonstrated positive staining (Figure 8A). Moreover, there was more obvious positive staining for the single- and dual-GF-loaded nanocomplex groups compared with the blank nanocomplex group. While the BMP-2-loaded nanocomplex group presented similar staining to that of the PlGF-2-loaded nanocomplex group, the dual-GF-loaded nanocomplex group presented more prominent positive staining, implying a greater potential in osteoinduction. The quantitative results of calcium deposition (Figure 8B) also confirmed the same trend. Collectively, our results suggest that the PlGF-2/BMP-2 immobilized on heparin-based nanocomplexes is effective in inducing osteogenic differentiation of MC3T3-E1 cells.

According to the results obtained from measuring CCK-8 activity, observing cell morphology, counting cell number, and measuring ALP activity and calcium deposition, the activities of the medium released with PlGF-2 and BMP-2 was essentially the same. The mechanism of 


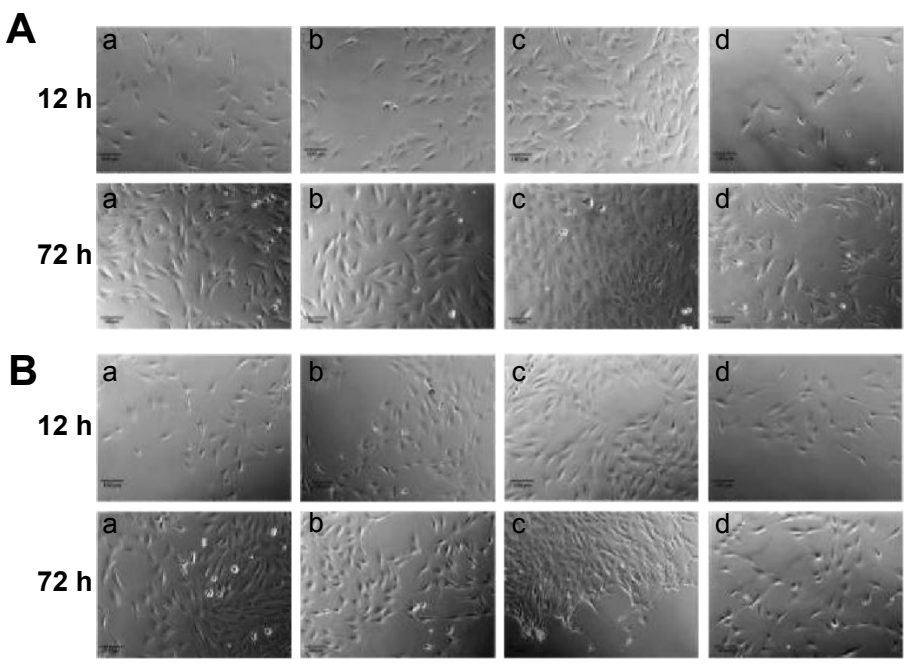

C

BMP-2-loaded heparin-nanocomplexes 㽞 PIGF-2-loaded heparin-nanocomplexes Z2 Dual-GF-loaded heparin-nanocomplexes DV Blank heparin-nanocomplexes

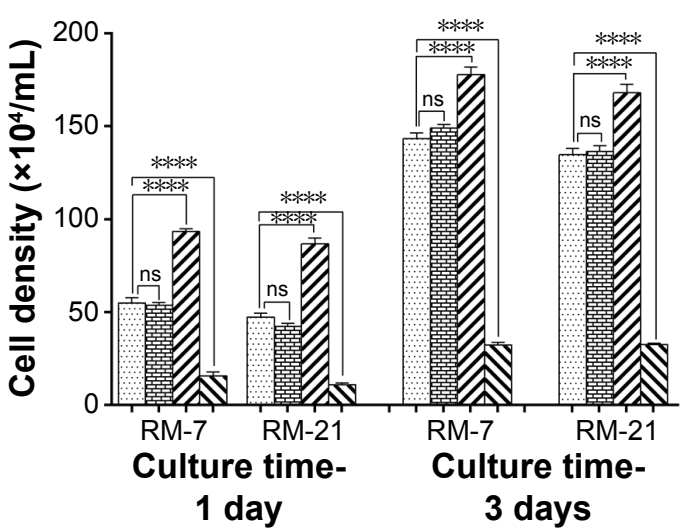

Figure 6 Microphotograph of MC3T3-EI cells after 12 and 72 hours of culturing with released medium collected on various nanocomplexes, as observed by using the inverted phase-contrast microscope.

Notes: The released medium was collected on days 7 (A) and 2 I (B). (a) BMP-2-loaded heparin-nanocomplexes; (b) PIGF-2-loaded heparin-nanocomplexes; (c) dual-GFloaded heparin-nanocomplexes; (d) blank heparin-nanocomplexes. The images were obtained at the same magnification $(\times 10)$. Scale bar $=100 \mu \mathrm{m}$. $(\mathbf{C}) \mathrm{Quantitative}$ analysis of MC3T3-EI cells after I and 3 days of culturing with various released mediums. ns $>0.05, * * * * P<0.000 \mathrm{I}$.

Abbreviations: BMP-2, bone morphogenetic protein; PIGF-2, placental growth factor-2; GF, growth factor; RM, released medium; RM-7, released medium collected on the seventh day; RM-2I, released medium collected on the 2 Ist day.

the osteogenic effects is complex, mainly owing to the reversible, noncovalent interactions between heparin and heparin-binding GFs, which ensure minimal impact on GF structure. ${ }^{13,30,37,39,43,44,48-52}$ We demonstrated that the nanocomplexes in our study not only effectively loaded and sustained release of PIGF-2/BMP-2, but also maintained their bioactivities for bone regeneration.

A key result in this study was the bioactivity of the released PIGF-2 being comparable to that of the released BMP-2, despite its dose being half that of BMP-2. According to the GF-loading efficiency coupled with the release profile, the PIGF-2 concentration in the released medium was slightly more than half of the BMP-2 concentration in the released medium. This phenomenon is supported by some recent research suggesting that PlGF-2 plays the role of a multitasking cytokine that stimulates bone regeneration by direct and indirect mechanisms. ${ }^{10,18}$ Apart from its traditional indirect role as an angiogenic factor, PlGF-2 also plays the unexpected role of a direct osteogenic factor. ${ }^{18}$ This latter role was first verified by Maes et al, ${ }^{18}$ who showed that PlGF-2 mediated the proliferation and osteoblastic differentiation of progenitor cells. Based on that research, a more recent study defined a pro-osteogenic mechanosensitive role for PIGF-2 in orchestrating osteogenic differentiation. ${ }^{10}$ According to that study, PIGF-2 works in a concentrationdependent manner. However, compared to BMP-2, PlGF-2 enhances osteogenic differentiation at lower concentrations and enhances vascularization at higher concentrations. The mechanism of this phenomenon may partly involve the extraordinarily promiscuous and strong binding of PlGF-2 to the extracellular matrix, which would orchestrate the availability and signaling of GFs. ${ }^{44}$ This phenomenon is

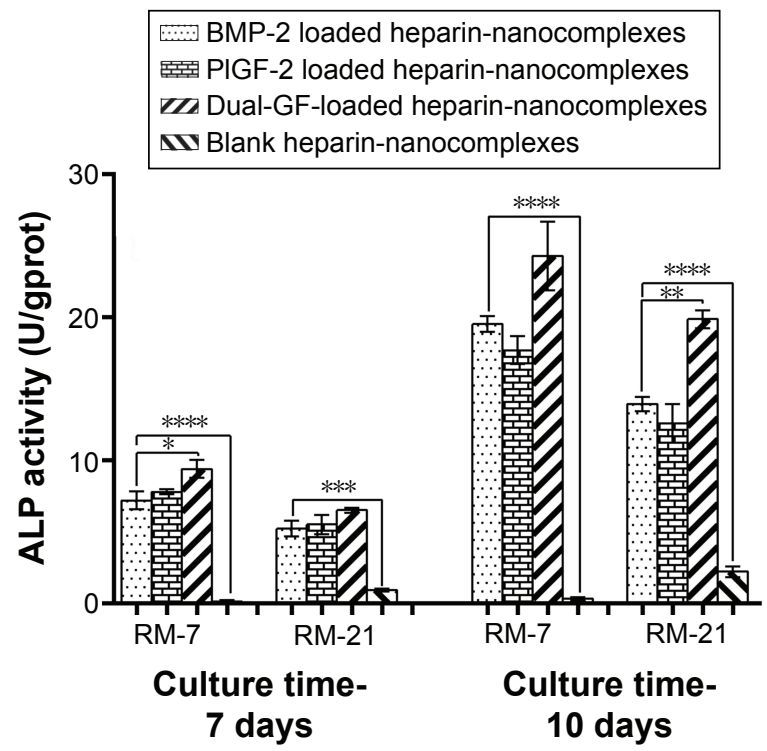

Figure 7 ALP activity of MC3T3-EI cells cultured with released mediums from different nanocomplexes for 7 and 10 days, retrospectively.

Notes: The released mediums were collected on days 7 and 21. Error bars represent SD. $* P<0.05, * * P<0.01$, and $* * * P<0.001$.

Abbreviations: BMP-2, bone morphogenetic protein; PIGF-2, placental growth factor-2; GF, growth factor; RM, released medium; RM-7, released medium collected on the 7th day; RM-2I, released medium collected on the 21 st day; SD, standard deviation. 

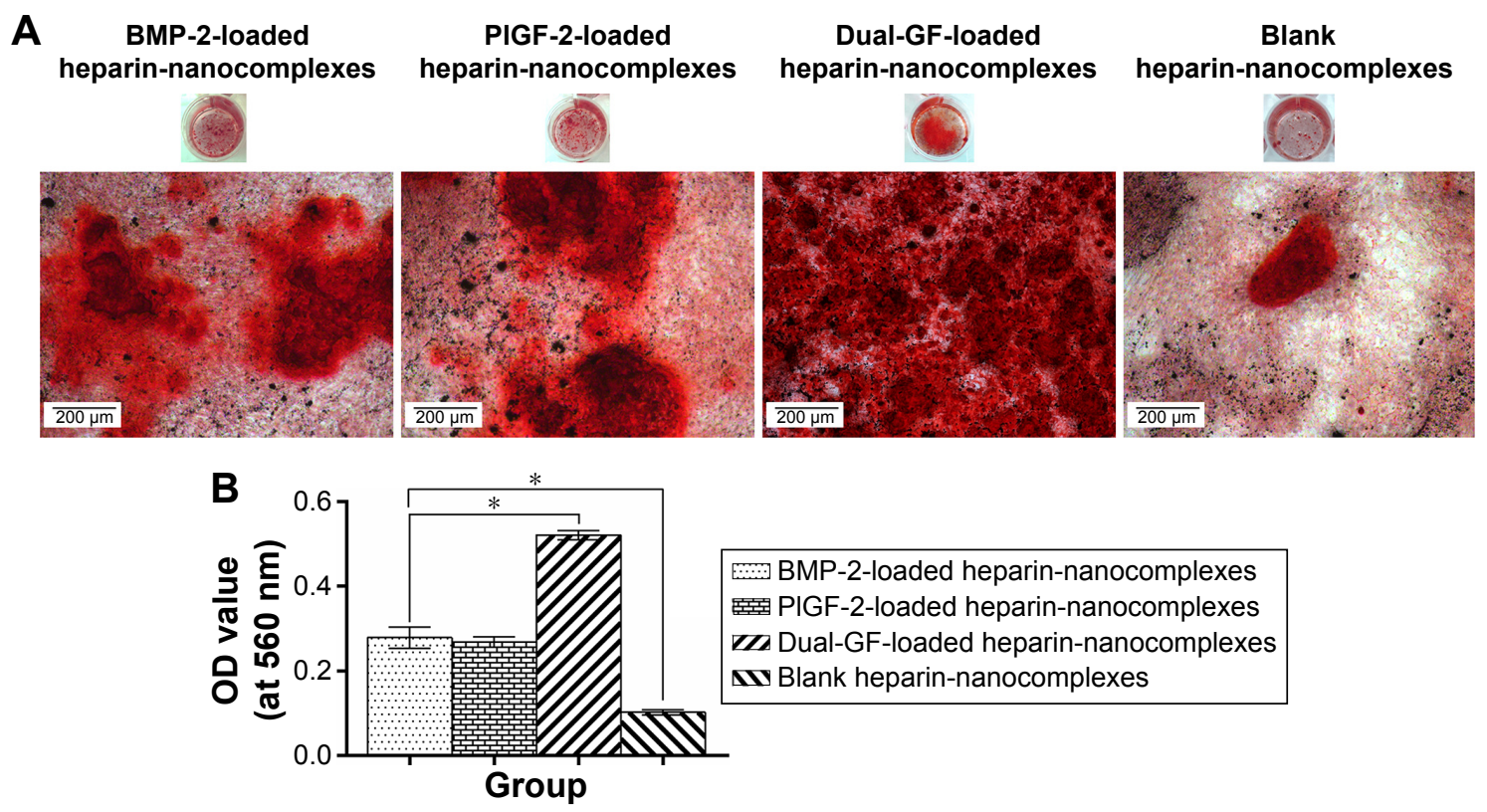

Figure 8 (A) ARS staining of MC3T3-EI cells cultured for 21 days in osteogenic induction microenvironments containing different released mediums. (B) Quantification of mineral deposition by detecting absorbance of ARS extracts.

Notes: Scale bar $=200 \mu \mathrm{m}$. Error bars represent SD. $* P<0.05$.

Abbreviations: BMP-2, bone morphogenetic protein; PIGF-2, placental growth factor-2; GF, growth factor; OD, optical density; ARS, Alizarin Red S; SD, standard deviation.

attractive for clinicians, as the current research typically focuses on BMP-2, which has undesirable side effects due to the large dose needed for achieving a therapeutic effect. Intriguingly, when PlGF-2 plays the role of a pro-osteogenic growth factor to enhance osteogenic differentiation, it requires a much lower concentration than that required for BMP-2. This therapeutic equivalence gives the clinician another choice. Upon comparing the osteogenic activity of the nanocomplexes loaded with the half-dose of PlGF-2 to that of the nanocomplexes loaded with the full-dose of BMP-2, the advantages of using PlGF-2 to avoid high costs and side effects become apparent.

The other key result in this study was the greater potential in direct osteogenesis for the dual delivery of PIGF-2 and BMP-2. In this study, the dual-GF-loaded nanocomplexes exhibited significantly higher activity than did the single-GFloaded nanocomplexes. The underlying cellular and molecular mechanisms are still complicated and elusive. According to Ramasamy et al, ${ }^{6}$ there is a molecular framework coupling osteogenesis and angiogenesis. Until recently, little was known about the normal function of PlGF-2 as an angiogenic factor. ${ }^{17}$ However, the current research suggests that PlGF-2 could be expressed by a series of cell types, including progenitors of the bone marrow. ${ }^{6,18-20}$ Maes et al ${ }^{18}$ indicated that the expression of PlGF-2 and its receptor increased throughout the repair process, and they suggested that PlGF- 2 coordinates the key aspects of bone repair. Moreover, PIGF-2 receptors, including neuropilin-2, are also expressed in osteoblast lineage cells during the repair of fractures, indicating a potent "switch" role of PlGF-2 in the process of osteogenesis. ${ }^{19}$ The key role of PlGF-2 in the osteogenesis/angiogenesis crosstalking framework could also be explained by its predominant role as the angiogenic and inflammatory "switch". ${ }^{19,20,53}$ Considering that the bone remodeling process is mediated by a series of inflammatory factors, it is reasonable to believe that PIGF-2 may play a key role in the osteogenesis/angiogenesis crosstalking framework. It can be concluded that there is an as yet unidentified molecular framework coupling osteogenesis and angiogenesis, and PlGF-2 plays key roles in such a framework. Just as the angiogenic activity of VEGF (the homologue of PlGF-2) could be amplified by combining it with BMP-2, ${ }^{53,54}$ our data also emphasized that the osteogenic activity of BMP-2 may be amplified by combining it with PIGF-2. Future work will be to explore the mechanism and develop an animal model to investigate the in vivo osteogenic effectiveness of PlGF-2/BMP-2-loaded nanocomplexes.

\section{Conclusion}

In this study, we used heparin-HTCC nanocomplexes to deliver PIGF-2 and BMP-2 for in vitro bone regeneration. Both GFs exhibited high loading efficiencies and sustained release. Compared with BMP-2, PlGF-2 enhanced osteogenic differentiation at a much lower loaded lose. Moreover, the osteogenic activity of BMP-2 may be amplified by combining it with PlGF-2. The dual delivery of PlGF-2 and BMP-2 could be used to improve osteoregeneration. 


\section{Acknowledgments}

This study was supported by the Natural Science Foundation of Guangdong Province, People's Republic of China (S2013010015805), as well as Guangdong Science and Technology Projects (2014A010105025). We thank all the research staff members at the Department of Oral Implantology, Guanghua School of Stomatology and the Institute of Polymer Science, Sun Yat-sen University.

\section{Disclosure}

The authors report no conflicts of interest in this work.

\section{References}

1. Cao L, Wang J, Hou J, Xing W, Liu C. Vascularization and bone regeneration in a critical sized defect using 2-N,6-O-sulfated chitosan nanoparticles incorporating BMP-2. Biomaterials. 2014;35(2):684-698.

2. Shah NJ, Hyder MN, Quadir MA, et al. Adaptive growth factor delivery from a polyelectrolyte coating promotes synergistic bone tissue repair and reconstruction. Proc Natl Acad Sci U S A. 2014;111(35): 12847-12852.

3. Cipitria A, Wagermaier W, Zaslansky P, et al. BMP delivery complements the guiding effect of scaffold architecture without altering bone microstructure in critical-sized long bone defects: a multiscale analysis. Acta Biomater. 2015;9(23):282-294.

4. Kusumbe AP, Ramasamy SK, Adams RH. Coupling of angiogenesis and osteogenesis by a specific vessel subtype in bone. Nature. 2014; 507(7492):323-328.

5. Novosel EC, Kleinhans C, Kluger PJ. Vascularization is the key challenge in tissue engineering. Adv Drug Deliv Rev. 2011;63(4-5):300-311.

6. Ramasamy SK, Kusumbe AP, Wang L, Adams RH. Endothelial Notch activity promotes angiogenesis and osteogenesis in bone. Nature. 2014; 507(7492):376-380.

7. Lee M, Li W, Siu RK, et al. Biomimetic apatite-coated alginate/chitosan microparticles as osteogenic protein carriers. Biomaterials. 2009; 30(30):6094-6101.

8. Cao L, Werkmeister JA, Wang J, Glattauer V, McLean KM, Liu C. Bone regeneration using photocrosslinked hydrogel incorporating rhBMP-2 loaded 2-N, 6-O-sulfated chitosan nanoparticles. Biomaterials. 2014;35(9):2730-2742.

9. Vo TN, Kasper FK, Mikos AG. Strategies for controlled delivery of growth factors and cells for bone regeneration. Adv Drug Deliv Rev. 2012;64(12):1292-1309.

10. McCoy RJ, Widaa A, Watters KM, et al. Orchestrating osteogenic differentiation of mesenchymal stem cells - identification of placental growth factor as a mechanosensitive gene with a pro-osteogenic role. Stem Cells. 2013;31(11):2420-2431.

11. Ben-David D, Srouji S, Shapira-Schweitzer K, et al. Low dose BMP-2 treatment for bone repair using a PEGylated fibrinogen hydrogel matrix. Biomaterials. 2013;34(12):2902-2910.

12. Rahman CV, Ben-David D, Dhillon A, et al. Controlled release of BMP-2 from a sintered polymer scaffold enhances bone repair in a mouse calvarial defect model. J Tissue Eng Regen Med. 2014;8(1):59-66.

13. Lo KW, Ulery BD, Ashe KM, Laurencin CT. Studies of bone morphogenetic protein-based surgical repair. Adv Drug Deliv Rev. 2012; 64(12):1277-1291.

14. Carmeliet $P$, Jain RK. Molecular mechanisms and clinical applications of angiogenesis. Nature. 2011;473(7347):298-307.

15. Fischer C, Jonckx B, Mazzone M, et al. Anti-PlGF inhibits growth of VEGF(R)-inhibitor-resistant tumors without affecting healthy vessels. Cell. 2007;131(3):463-475.

16. Jain RK, Xu L. $\alpha$ PIGF: a new kid on the antiangiogenesis block. Cell. 2007;131(3):443-445.
17. Ribatti D. The discovery of the placental growth factor and its role in angiogenesis: a historical review. Angiogenesis. 2008;11(3):215-221.

18. Maes C, Coenegrachts L, Stockmans I, et al. Placental growth factor mediates mesenchymal cell development, cartilage turnover, and bone remodeling during fracture repair. J Clin Invest. 2006;116(5): $1230-1242$.

19. Fischer C, Mazzone M, Jonckx B, Carmeliet P. FLT1 and its ligands VEGFB and PlGF: drug targets for anti-angiogenic therapy? Nat Rev Cancer. 2008;8(12):942-956.

20. Dewerchin M, Carmeliet P. PlGF: a multitasking cytokine with diseaserestricted activity. Cold Spring Harb Perspect Med. 2012;2(8):1-25.

21. Marrony S, Bassilana F, Seuwen K, Keller H. Bone morphogenetic protein 2 induces placental growth factor in mesenchymal stem cells. Bone. 2003;33(3):426-433.

22. Jacobsen KA, Al-Aql ZS, Wan C, et al. Bone formation during distraction osteogenesis is dependent on both VEGFR1 and VEGFR2 signaling. J Bone Miner Res. 2008;23(5):596-609.

23. Otomo H, Sakai A, Uchida S, et al. Flt-1 tyrosine kinase-deficient homozygous mice result in decreased trabecular bone volume with reduced osteogenic potential. Bone. 2007;40(6):1494-1501.

24. Koichi HB, Yan W, Sergio D, et al. Placental growth factor reconstitutes hematopoiesis by recruiting VEGFR 1 (+) stem cells from bone-marrow microenvironment. Nat Med. 2002;8(8):841-849.

25. Street J, Lenehan B. Vascular endothelial growth factor regulates osteoblast survival - evidence for an autocrine feedback mechanism. J Orthop Surg Res. 2009;4(19):1-13.

26. Niida S, Kondo T, Hiratsuka S, et al. VEGF receptor 1 signaling is essential for osteoclast development and bone marrow formation in colonystimulating factor 1 - deficient mice. Proc Natl Acad Sci U S A. 2005; 102(39):14016-14021.

27. Henriksen K, Karsdal M, Delaisse JM, Engsig MT. RANKL and vascular endothelial growth factor (VEGF) induce osteoclast chemotaxis through an ERK1/2 - dependent mechanism. J Biol Chem. 2003;278(49): 48745-48753.

28. Maes CC, Schipani E. Hypoxia-driven pathways in bone development, regeneration and disease. Nat Rev Rheumatol. 2012;8(6):358-366.

29. Jaepyoung CJ, Micheline PM, Christine A. Synthesis and physicochemical and dynamic mechanical properties of a water-soluble chitosan derivative as a biomaterial. Biomacromolecules. 2006;7(10): 2845-2855.

30. Wang B, Tan L, Deng D, et al. Novel stable cytokine delivery system in physiological $\mathrm{pH}$ solution: chitosan oligosaccharide/heparin nanoparticles. Int J Nanomedicine. 2015;10:3417-3427.

31. Zhou X, Wei F, Qiu K, et al. BMP-2 derived peptide and dexamethasone incorporated mesoporous silica nanoparticles for enhanced osteogenic differentiation of bone mesenchymal stem cells. ACS App Mater Interfaces. 2015;7(29):15777-15789.

32. Liang C, Wang H, Yang J, et al. Femtosecond laser-induced micropattern and $\mathrm{Ca} / \mathrm{P}$ deposition on Ti implant surface and its acceleration on early osseointegration. ACS Appl Mater Interfaces. 2013;5(16): 8179-8186.

33. Zhuang XM, Zhou B, Ouyang JL, et al. Enhanced MC3T3-E1 preosteoblast response and bone formation on the addition of nano-needle and nano-porous features to microtopographical titanium surfaces. Biomed Mater. 2014;9(4):1-11.

34. Carl AG, Grady G, Alexandra P, Darwin JP. An Alizarin red-based assay of mineralization by adherent cells in culture: comparison with cetylpyridinium chloride extraction. Anal Biochem. 2004;329(1):77-84.

35. Zuzana S, Eric H, Caroline M, Alain B, Pierre JM. Strontium ranelate rebalances bone marrow adipogenesis and osteoblastogenesis in senescent osteopenic mice through NFATcMaf and Wnt signaling. Aging Cell. 2012;11(3):467-474.

36. Lu LX, Zhang XF, Wang YY, et al. Effects of hydroxyapatite-containing composite nanofibers on osteogenesis of mesenchymal stem cells in vitro and bone regeneration in vivo. ACS Appl Mater Interfaces. 2013; 5(2):319-330. 
37. Abbah SA, Liu J, Lam RW, Goh JC, Wong HK. In vivo bioactivity of rhBMP-2 delivered with novel polyelectrolyte complexation shells assembled on an alginate microbead core template. J Control Release. 2012;162(2):364-372.

38. Place ES, Evans ND, Stevens MM. Complexity in biomaterials for tissue engineering. Nat Mater. 2009;8(6):457-470.

39. Murali S, Rai B, Dombrowski C, et al. Affinity-selected heparan sulfate for bone repair. Biomaterials. 2013;34(22):5594-5605.

40. Martino MM, Briquez PS, Ranga A, Lutolf MP, Hubbell JA. Heparinbinding domain of fibrin(ogen) binds growth factors and promotes tissue repair when incorporated within a synthetic matrix. Proc Natl Acad Sci US A. 2013;110(12):4563-4568.

41. Tan Q, Tang H, Hu J, et al. Controlled release of chitosan/heparin nanoparticle-delivered VEGF enhances regeneration of decellularized tissue-engineered scaffolds. Int J Nanomedicine. 2011;6:929-942.

42. Almodovar J, Kipper MJ. Coating electrospun chitosan nanofibers with polyelectrolyte multilayers using the polysaccharides heparin and N,N,N-trimethyl chitosan. Macromol Biosci. 2011;11(1):72-76.

43. Bramono DS, Murali S, Rai B, et al. Bone marrow-derived heparan sulfate potentiates the osteogenic activity of bone morphogenetic protein-2 (BMP-2). Bone. 2012;50(4):954-964.

44. Martino MM, Briquez PS, Guc E, et al. Growth factors engineered for super-affinity to the extracellular matrix enhance tissue healing. Science. 2014;343(6173):885-888.

45. Stegen S, van Gastel N, Carmeliet G. Bringing new life to damaged bone: the importance of angiogenesis in bone repair and regeneration. Bone. 2015;70:19-27.
46. Wang M, Feng Q, Guo X, She Z, Tan R. A dual microsphere based on PLGA and chitosan for delivering the oligopeptide derived from BMP-2. Polym Degrad Stab. 2011;96(1):107-113.

47. Kim SE, Yun YP, Lee JY, Shim JS, Park K, Huh JB. Co-delivery of platelet-derived growth factor (PDGF-BB) and bone morphogenic protein (BMP-2) coated onto heparinized titanium for improving osteoblast function and osteointegration. J Tissue Eng Regen Med. 2013;9(12):219-228

48. Hu L, Sun Y, Wu Y. Advances in chitosan-based drug delivery vehicles. Nanoscale. 2013;5(8):3103-3111.

49. Amidi M, Mastrobattista E, Jiskoot W, Hennink WE. Chitosan-based delivery systems for protein therapeutics and antigens. Adv Drug Deliv Rev. 2010;62(1):59-82.

50. Yang Y, Wang S, Wang Y, Wang X, Wang Q, Chen M. Advances in self-assembled chitosan nanomaterials for drug delivery. Biotechnol Adv. 2014;32(7):1301-1316.

51. Jiang BH, Chen WY, Li HY, et al. CHD1L regulated PARP1-driven pluripotency and chromatin remodeling during the early-stage cell reprogramming. Stem Cells. 2015;33(10):2961-2972.

52. Santos MI, Reis RL. Vascularization in bone tissue engineering: physiology, current strategies, major hurdles and future challenges. Macromol Biosci. 2010;10(1):12-27.

53. Bai Y, Leng Y, Yin G, et al. Effects of combinations of BMP-2 with FGF-2 and/or VEGF on HUVECs angiogenesis in vitro and CAM angiogenesis in vivo. Cell Tissue Res. 2014;356(1):109-121.

54. Richard AD, Ellen HF. Angiogenesis and bone repair. Drug Discov Today. 2003;8(21):980-989. 


\section{Supplementary material}
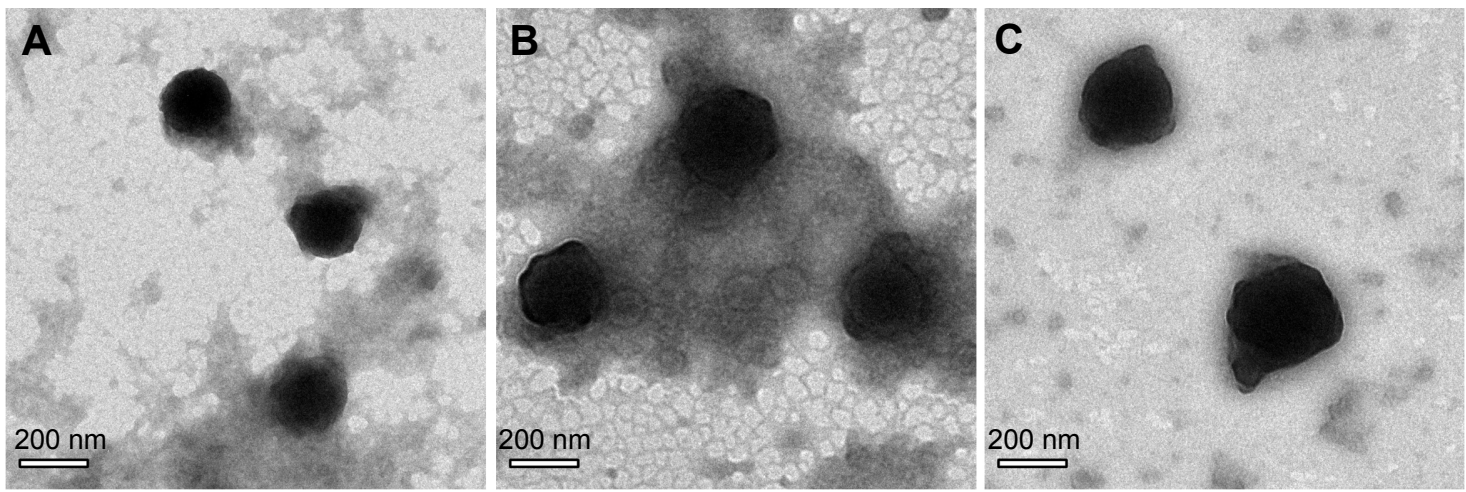

Figure SI TEM images of heparin-HTCC nanocomplexes before and after PIGF-2/BMP-2/dual-GF loading.

Notes: (A) Blank heparin-nanocomplexes; (B) BMP-2-loaded heparin-nanocomplexes; (C) PIGF-2-loaded heparin-nanocomplexes (scale bar =200 nm).

Abbreviations: TEM, transmission electron microscopy; HTCC, N-(2-hydroxyl)propyl-3-trimethyl ammonium chitosan chloride; PIGF-2, placental growth factor-2; BMP-2, bone morphogenetic protein; GF, growth factor.

\section{Publish your work in this journal}

The International Journal of Nanomedicine is an international, peerreviewed journal focusing on the application of nanotechnology in diagnostics, therapeutics, and drug delivery systems throughout the biomedical field. This journal is indexed on PubMed Central, MedLine, CAS, SciSearch $®$, Current Contents ${ }^{\circledR} /$ Clinical Medicine,
Journal Citation Reports/Science Edition, EMBase, Scopus and the Elsevier Bibliographic databases. The manuscript management system is completely online and includes a very quick and fair peer-review system, which is all easy to use. Visit http://www.dovepress.com/ testimonials.php to read real quotes from published authors. 